\title{
LA RECEPCIÓN DEL QUIJOTE EN LOS SIGLOS XVII Y XVIII EN TIERRAS ALEMANAS, ¿'BIEN' O 'HERRAMIENTA'?
}

ARTURO PARADA

Universidade de Vigo

\section{INTRODUCCIÓN}

Pretendo en este trabajo trazar un esbozo de la recepción del Quijote en tierras alemanas en los siglos XVII y XVIII atendiendo a una diferenciación esencial dentro de la teoría de los polisistemas. La suerte que el Quijote corrió en Alemania y la contribución de la novela de Cervantes al nacimiento de la novela alemana en general, de la novela, en términos bajtinianos, polifónica en particular está analizada por extenso en Parada, 1997.

\section{BIENES Y HERRAMIENTAS}

La distinción que establece Even-Zohar entre bienes y herramientas en/de una cultura es conocida ${ }^{1}$; antes de realizar una re-

\footnotetext{
${ }^{1}$ No es este el momento de entrar en la muy amplia, complicada y, mientras tanto, en ocasiones 'barroca' discusión de lo que determina y constituye el término de 'cultura', y si bien las 'Kulturwissenschaften', las ciencias de la cultura, dicen haber solucionado la cuestión (cf. por ejemplo los trabajos de Hartmut Böhme), un trabajo de recopilación, panorámico, como el de Swingewood (Swingewood, 1998) subraya, una vez más, que el concepto de cultura está, naturalmente, muy ligado a ideología, y sujeto, por tanto, al grado de variabilidad que la misma relación lleva implícita. Trabajos recientes del campo de la translatología, de las filologías interculturales, en proceso de definición y constitución, o de la literatura comparada demuestran que, si bien el término se ha hecho ya imprescindible, este ha de seguir respondiendo a la flexibilidad que la ocasión requiere, lo cual sigue constituyendo, hoy por hoy, su característica más predominante (para el desarrollo de la translatología cf. Neubert, 1999; para la filología intercultural

AC. XXXVI (2004), 345-356
} 
capitulación quiero adelantar que si Even-Zohar considera, con Renfrew, que es más fácil que los bienes pasen a convertirse en herramientas que a la inversa, la recepción del Quijote en Alemania ofrece ejemplos claros de ambos procesos, de modo que la 'herramienta Quijote', convenientemente 'manejada' y aprovecha$\mathrm{da}$, acaba convirtiéndose, efectivamente, en bien, y este, en herramienta, aunque, como veremos, no con el resultado esperado o deseado. Estos procesos de asimilación de literatura universal presentan, desde luego, aspectos paradigmáticos.

Según Even-Zohar (1999 [1997]: 27-36), los bienes de una cultura son aquellos que poseen un 'valor', esto es, que pueden ser evaluables, para lo cual es condición inexcusable que exista un «mercado acreditado». Para épocas muy antiguas, Even-Zohar no parte, sin embargo, de lo colectivo, sino de lo individual: «De un estado en el cual para ser evaluables deben pertenecer a un gobernante, se transforman en bienes que pertenecen "a todos" " (1999:30). El paso descrito en la apreciación de los bienes iría, pues, de lo individual a lo colectivo, si bien la historia de occidente se caracteriza por un fuerte proceso, interrumpido en ocasiones, de individualización, lo cual equivale a decir que es la singularidad o particularidad la que parece va cobrando peso, también, precisamente, entre la colectividad (cf. Elías, 1990; Hauser, 1988; Parada, 2003) ${ }^{2}$. Es esta una contradicción que no se resuelve con facilidad, pues se trata de dilucidar quién o qué marca establece de hecho las pautas artísticas en las diversas sociedades y en los distintos momentos históricos. Claudio Guillén nos ha enseñado a este respecto que, por muy grandes que puedan llegar a ser las diferencias entre los países en cuanto a su configuración social, los conflictos entre el poder político y el artista siempre han estado a la orden del día y que, por tanto, la balanza del 'poder artístico' nunca se ha inclinado claramente hacia uno de los lados (cf. Guillén, 1995). Las culturas - naciones a veces- que entran

cf. en general los trabajos de Alois Wierlacher, para la literatura comparada cf. el reciente Gnisci, 2002, especialmente los capítulos VIII, "La literatura y los estudios interculturales» y IX, "Multiculturalismo, estudios poscoloniales y descolonización»). De este modo, cultura se 'va definiendo' en la misma medida en que las diversas disciplinas echan mano del concepto para llegar a observaciones/conclusiones más amplias y, a su vez, más ajustadas. La bibliografía al respecto es, mientras tanto, inabarcable; precisas, actualizadas y sintéticas definiciones de los conceptos de cultura, antropología cultural, ciencias de la cultura, transferencia cultural, etc., etc. en relación con la literatura y las ciencias literarias se encuentran en Nünning, 2001.

${ }^{2}$ No es este el momento de entrar en la discusión en torno al concepto de originalidad en relación con el arte occidental, particularmente por lo que a la Edad Media se refiere 
en el siglo XVIII en el proceso de diferenciación funcional percibirán cómo se van definiendo y especificando ámbitos - 'campos' en la terminología de Bourdieu- y, con ello, las correspondientes estructuras, con sus veedores, enjuiciadores y prefectos ${ }^{3}$. El mismo Even-Zohar subraya insistentemente la importancia de estas relaciones cambiantes de poder, que, como veremos, desempeñan también en la recepción del Quijote un papel fundamental.

Herramientas en/de una cultura son aquellas que sirven para 'organizar' la vida, tanto a un nivel individual como colectivo, distinguiéndose herramientas pasivas, de cualidad preferentemente hermenéutica e intelectual, y herramientas activas, que constituyen la vertiente aplicada, práctica, en un sentido pleno.

Importante es, con todo, no olvidar que, háblese de bienes o de herramientas, la teoría de los sistemas atiende el hecho literario no como objeto o 'producto' artístico aislado o autosuficiente, sino en su totalidad, esto es, teniendo en cuenta todos y cada uno de los factores involucrados en la producción, distribución, transmisión, valoración, etc., de literatura.

Nos encontramos, así, ante un enfoque que resulta provechoso cuando se pretende analizar causas, modos, procedimientos y resultados de la 'llegada' del Quijote a tierras alemanas, recepción en la que influyen un cúmulo de factores.

\section{ANTECEDENTES: EL QUIJOTE EN EL SIGLO XVII ALEMÁN}

La pronta recepción del Quijote en Inglaterra tiene más que ver con la existencia de una tradición irónico-dialógica y humanística que con la creación de un 'mercado literario', lo cual, en todo caso, reforzó y, en ocasiones, polarizó corrientes de pensamiento existentes; de esta forma, el elemento desmitificador, primero, el, digamos, reivindicativo después pudo enriquecer como quien dice de forma 'natural' formas de pensamiento previamente existentes y de larga tradición (cf. para la tradición dialógica inglesa y la recepción del Quijote Parada, 1997:129-147). El famoso debate Wilkins/ Ward-Webster demuestra a la perfección cómo el Quijote, tan pronto como aparece en el ámbito cultural inglés, se convierte de inmediato en una herramienta con el suficiente 'valor' acumulado como para representar un arma temible. Se da, así, el caso sin-

\footnotetext{
${ }^{3}$ Se trata de un proceso que no afecta, naturalmente, a todos los países europeos por igual y que, en todo caso, parece desarrollarse de forma más decidida allí en donde se impone la reforma protestante y un cierto espíritu racionalista. En este sentido, no faltan estudiosos que sitúan los comienzos de esta autonomización en el Renacimiento (así Jurt, 1997).
} 
gular de que hay dificultades para juzgar si la herramienta es tan efectiva porque se trata de un bien con el suficiente prestigio - "His predecesor in the Military way the famous Hero of the Mancha», afirman, jen 1654!, Wilkins y Ward refiriéndose a su contrincante Webster (Debus, 1970:204) - o si, por el contrario, es la calidad de la herramienta la que confiere al elemento cultural en cuestión de forma casi inmediata el estatus o la categoría de bien, pues ya en Ben Jonson se detectan, asimismo, a principios del siglo XVII reminiscencias de la novela de Cervantes (cf. Mahler, 1991) .

Si dejamos al margen una aparición anecdótica del hidalgo manchego en Alemania (en 1613, con ocasión de una parodia de los torneos caballerescos), y las breves observaciones que sobre el Quijote hacen Harsdörffer y Rist, el primero que 'utiliza' la novela de Cervantes es Christian Thomasius, profesor universitario, jurista, filósofo, temido polemista y convencido protestante que intenta rescatar, frente a la tradición escolástica y cierto espíritu 'agelasta' (esto es, enemigo de la risa), el humanismo de tinte erasmista para la Universidad y las Iglesias reformadas y, con ello, para Alemania.

El caso de Thomasius demuestra a las claras cómo se puede hacer uso de una 'herramienta cultural' mucho antes de que esta se haya convertido en bien, pues de la misma manera que Thomasius no duda en exponer y comentar el Oráculo manual de Gracián con el fin de favorecer el ascenso social de sus estudiantes, así tampoco teme ayudarse de literatos extranjeros, carentes todavía de prestigio o plena aceptación social no sólo sus obras, sino también el oficio mismo al que se dedican, especialmente en las culturas en las que se han impuesto las Iglesias reformadas: baste pensar en las furibundas diatribas que lanza el pastor suizo Gotthard Heidegger en su Mythoscopia Romantica oder Discours von den so benanten Romans, Zúrich, 1698, [Mythoscopia Romántica o discurso sobre la así llamada novela] contra la novela ${ }^{5}$. En los países de lengua alemana, el género, la 'forma' según otros, novela tendrá que esperar hasta la segunda mitad del siglo XVIII para poder matizar o menguar esa fama de quimera, desvarío, que lo acompaña prácticamente desde su nacimiento, si bien se multiplicarán entonces las voces que reclamen, con el fin de ponerse a la altura de las demás lenguas y naciones europeas, la 'novela alemana'.

\footnotetext{
${ }^{4}$ Contamos con un trabajo reciente, que no he podido consultar, sobre la relación literaria Cervantes-Ben Jonson: Yumiko YAmadA, Ben Jonson and Cervantes: tilting against chivalric romances, Maruzen, Tokio, 2000.

${ }^{5}$ Existe una edición facsimilar de 1969, editada por Ernst Schäfer.
} 
Tanto más llamativo y sorprendente resulta, por tanto, que Thomasius, un, no se olvide, primer ilustrado, haga una lectura lo suficientemente 'abierta' del Quijote como para recurrir a la novela de Cervantes de forma manifiesta y explícita, tomando la obra como modelo ético y estético, y ello en este orden, con el fin de promover la crítica y el diálogo social a favor del bien común, en contra de estructuras escolásticas anquilosadas y en pro de una sociedad más abierta que aquella que le tocó vivir, guiándose por el lema de san Pablo: "porque la letra mata, mientras que el espíritu da vida" (2 Cor. 3:6) (cf. para el binomio literatura-académicos/humanistas alemanes el magnífico trabajo de Grimm, 1983; para lo que nos concierne, cf. Parada, 1997:112-129).

En manos de Thomasius, la novela, y la figura, ideada por Cervantes ejerce, pues, de herramienta mucho antes de que el Quijote se convierta, asimismo, en un muy preciado bien para la cultura alemana.

\section{El QuiJote EN EL SIgLO XVIII ALEMÁN, EL QUIJOTE POLARIZADO}

Las Moralische Wochenschriften, las revistas alemanas hechas en su mayoría a imagen y semejanza de las inglesas The Tatler y The Spectator, difunden entre una burguesía cada vez más amplia, cada vez más fuerte y progresivamente letrada las modas y corrientes literarias que llegan del extranjero, sobre todo, en un principio, de Inglaterra, donde, como queda dicho, el Quijote tiene una pronta y buena acogida. Sin embargo, incluso antes de que la polarización literaria que se da en Inglaterra en el siglo XVIII entre lectores partidarios de Samuel Richardson, por un lado, y de Henry Fielding, por otro, se traslade a otras culturas europeas, tienen lugar sobre tierras germano-suizas intensas controversias literarias, las cuales hay que contemplar ante el trasfondo de la apertura de un campo social y económico relativamente nuevo, el de la literatura, ahora más abierto y competitivo a la vez y desde un primer momento en estrecha relación con los demás ámbitos parciales. El objeto de estas discusiones será con frecuencia el Quijote, obra que, sin embargo, no sólo sirve como pretexto o punto de referencia para debatir cuestiones estéticas en torno al arte, en general, y la novela, género pujante, en particular, sino también para poner en tela de juicio asuntos que tienen que ver con la reestructuración de los órdenes sociales y la 'Entzauberung', 'desmagiación' del mundo.

Buen ejemplo de ello es la discusión, que ha entrado en la historia de la literatura alemana, que mantienen los suizos Bodmer 
y Breitinger con el alemán Gottsched, todos ellos influyentes críticos de su época, en torno a la validez de los modelos ingleses o franceses, sobre si cabe leer el Quijote de una manera o de otra y cuál es, en relación con ello, el papel que puede y debe concedérsele a la fantasía y a lo 'maravilloso' (cf. Parada, 1997:93-102). Mientras que los primeros se muestran partidarios de la 'realidad' como referente del arte, sin excluir por ello lo maravilloso, esto es, lo imaginado, Gottsched se inclina por los modelos 'estetizantes' de la comedia clásica francesa: lo que se dirime, pues, aquí no es sólo una concepción artística, sino el lugar que ha de corresponderle al arte y, naturalmente, al artista en sociedad.

Antagonista también de Gottsched, pero crítico asimismo con los suizos, admirador incondicional de Shakespeare, es Gotthold Ephraim Lessing (1729-1781), uno de los fundadores del teatro moderno alemán, quien parte de que a la literatura, en particular, cabe una función predominantemente social y, anticipándose en ello a Schiller, educativa, de modo que el artista ha de vivir para y también de su arte. Convencido, pues, de la importancia de la literatura, Lessing, que ve surgir necesidades y posibilidades sociales y literarias nuevas, pretende profesionalizar su vocación y vivir exclusivamente de lo que escribe, convirtiéndose así en el primer 'escritor' en un sentido moderno del término de la historia de la literatura en lengua alemana.

Dado que no lo consigue, Lessing se ve obligado a trabajar los últimos años de su vida como bibliotecario en la Herzog August Bibliothek de Wolfenbüttel, biblioteca importante ya para su época y que guarda hoy un fondo notable de primeras y segundas ediciones de obras cervantinas en diversos idiomas, el español entre ellos (puede consultarse el catálogo en http://www.hab.de/). Si se analizan las estadísticas de préstamos que se realizaron entre 1714 y 1799 en esta biblioteca, podemos observar que el Quijote es uno de los libros más solicitados, y ello sin que figuren entre los títulos obras de Fielding o Sterne (cf. Raabe, 1989; Parada, 1997: 147 ss.), lo cual obliga a modificar en un sentido inverso la extendida y aceptada teoría que afirma que fueron las novelas de Fielding y Sterne las que favorecieron la propagación del Quijote por tierras alemanas: fue el amplio conocimiento que el público lector alemán tenía ya hacia 1740 del Quijote el que facilitó la excelente acogida de las obras de estos autores. Esto explica también por qué en las Moralische Wochenschriften se encuentran una y otra vez alusiones y referencias al Quijote. Del análisis de estas revistas se concluye que si la lectura más común que se hace en la primera mitad del siglo XVIII de la obra está determinada por un espíritu racionalista-ilustrado, hacia 1750 es posible percibir ya 
un cambio o giro hacia una interpretación más idealista, benigna, sentimental si se quiere.

Hay que constatar, en todo caso, que hacia finales del siglo XVIII el Quijote se ha convertido ya definitivamente en un bien para la cultura alemana, polivalente además, que sirve tanto para denostar de las quimeras vanas como para reivindicar la aventura, la entrega apasionada en pro de un ideal. Interesante a este respecto es constatar cómo un bien puede entrar a formar parte de una cultura sin que exista todavía una entidad política superior, amplia, estabilizada, la cual, como es sabido, 'Alemania' no alcanza hasta finales del siglo XIX. Cambiante es también, en este sentido, la opinión que a partir del o en relación con el Quijote se crea de España y de los españoles en tierras alemanas, aunque prevalece frecuentemente la interpretación francesa según la cual Cervantes se había propuesto caricaturizar un defecto nacional: «Denn Don Quixote ist nichts anders, als eine symbolische Person, welche erfunden worden, eine besondere und merckwürdige Eigenschaft in dem Charakter der Spanischen Nation vor den Augen aller Welt zu spielen.», afirma el mismo Bodmer en su conocido ensayo "Von dem Charakter des don Quixote und Sancho Pansa» (cf. Parada, 1997:99-100) [«Pues no es don Quijote sino una figura simbólica inventada para mostrar ante los ojos del mundo una particularidad extraña en el carácter de la nación española». Traducción mía. A. P.].

La 'adquisición' del Quijote para el acervo cultural alemán permitirá entonces volver una y otra vez sobre la novela de Cervantes para reforzar la propia posición, quedando de esta manera confirmada, y matizada, la observación de Even-Zohar respecto a la importancia de las lenguas y literaturas nacionales -e internacionales, cabría entonces añadir-, para la construcción de una identidad 'nacional' (Even-Zohar, 1997:31). El caso alemán es, en este sentido, paradigmático y específico a la vez, pues ante la ausencia de una unidad política y geográfica, cobran importancia y se imponen los conceptos y las concepciones abstractas de 'Vaterland' [patria], 'patriotismo', 'nación', 'Deutsche Seele' [alma alemana], lengua, Kultur, 'Preußentum' [espíritu prusiano] y, sobre todo, las virtudes burguesas, sirviendo todo ello como sustituto balsámico, aglutinante y base para la construcción de la identidad y nación alemana (cf. Blitz, 2000:337 ss.). En esta tarea, la literatura desempeña un papel esencial, mas no completamente desinteresado: «Die Entdeckungen eines preußischen wie eines deutschen Vaterlandes als mögliche dichterische Themen werden von massiven Marktüberlegungen begleitet.» (Blitz, 2000:209) [«El descubrimiento de una patria prusiana y alemana como posibles temas litera- 
rios va acompañado de numerosos y notorios cálculos de mercado». Traducción mía, A. P.]

Cálculos de mercado, esto es, afán de éxito e intereses económicos, se esconden también tras dos de las primeras imitaciones del Quijote en lengua alemana.

\section{DOS INTENTOS DE CONVERTIR UN BIEN EN HERRAMIENTA: NEUGEBAUER Y WiELAND}

Wilhelm Ehrenfried Neugebauer es autor de Der teutsche Don Quichotte, Oder die Begebenheiten des Marggraf von Bellamontel Komisch und satyrisch beschrieben; aus dem Französischen übersetzt, Breslau 1753, esto es: El don Quijote alemán o los hechos del Margrave de Bellamonte, descritos de forma cómica y satírica, obra traducida del francés ${ }^{6}$, una novela que, de contenido tan convencional como artificioso, llamó en la década de 1960 la atención de algunos germanistas en cuanto estos creían ver en ella un narrador plural, múltiple, metanovelístico y, con ello, la tan ansiada innovación o modernidad novelística en la literatura alemana del siglo XVIII. Es cierto que en el Der teutsche Don Quichotte hay un narrador que dialoga con el lector, que va explicando este o aquel detalle de la trama, que inserta glosas y análisis. Hasta aquí llegan, sin embargo, las concomitancias con el modelo que sirvió de inspiración, pues lejos de fragmentar las perspectivas, el autor las reduce a una, la del narrador, que coincide, a su vez, la de un público burgués que Neugebauer sabe de acuerdo con la denuncia no ya de la novela cortesano-histórica y galante, sino de todo planteamiento o actitud que se aparte del «Mittelwege der gesunden Vernunft», del camino recto de la razón o del sentido común.

La novela de Christoph Martín Wieland, autor importante dentro de la literatura alemana, Der Sieg der Natur über die Schwärmerey oder Die Abentheuer des Don Sylvio von Rosalva, de 1764, (El triunfo de la naturaleza sobre el entusiasmo arrobado o Las aventuras de don Silvio von Rosalva) no difiere mucho, en cuanto a modelos y procedimientos, de la novela de Neugebauer: la acción se sitúa en una España fabulosa, casi delirante, los personajes, sus acciones, el espacio en que se mueven no pasan de ser de cartón-piedra. En este sentido, conviene recordar que el mismo Wieland no ocultó que, imitando un modelo ya consagra-

${ }^{6}$ Existe una edición facsimilar, Stuttgart 1971. Cf. PARADA, 1997:192-197 y p. 13, donde se encuentran las correspondientes referencias bibliográficas. 
do, pretendía mejorar su economía a la vez que distanciarse de un pasado personal guiado por un entusiasmo idealista. Para lograr lo uno y lo otro recurrió al patrón que mayor éxito prometía.

La reducida posibilidad de realizar distintas lecturas de estas novelas está motivada, dejando cuestiones de talento al margen, por el peso excesivo de consideraciones utilitaristas, que priman claramente por encima de los objetivos artísticos (cf. para ambas obras, Parada, 1997:192-197) ${ }^{7}$. Neugebauer hubiera querido convertirse en pedagogo y legitimarse como autor de novelas ante un público burgués, valiéndose para ello de procedimientos que no se correspondían ni con sus intenciones ni con la tradición ni con el momento cultural en el que se encontraba su público potencial, y que violentaban, además, desde el momento en que estas 'tácticas' se utilizaban de forma limitada, restringida o cerrada, el mismo género al que se estaba recurriendo, y si don Diego de Miranda se admiraba ante "un cuerdo loco y un loco que tiraba a cuerdo», en Der teutsche Don Quichotte no queda en absoluto espacio para la admiración que provoca un mundo no concluso, esto es, abierto.

El caso de Wieland es más complejo, pues estamos ante un autor de muy vasta cultura, admirador rendido de Shakespeare ante un escritor que sabía muy bien de la relatividad de opiniones y creencias y de la conveniencia de considerar, sin rechazar ninguna de manera definitiva, múltiples perspectivas. Sin embargo, el Don Sylvio sólo resulta convincente allí en donde su autor abandona el terreno, falso, del realismo y se adentra en el cuento fantasioso. A este respecto, no deja de resultar sintomático que Wieland leyese y 'estudiase' el Quijote de la mano de Johann Wilhelm Baumer, que, adepto de las teorías filosóficas de Christian Wolff, expone ante su por entonces joven discípulo al mismo tiempo y de forma sistemática las corrientes filosóficas de su época, prestando, claro está, atención especial a Leibniz (cf. Hinderer, 1993:187). Al cotejar, pues, el Don Sylvio con su modelo se adivina la dificultad que van a tener los escritores alemanes para ofrecer aquello que múltiples voces del siglo XVIII reclaman una y otra vez: un retrato, vivo, de las circunstancias alemanas.

\footnotetext{
${ }^{7}$ Hay también valoraciones muy positivas de la novela de Wieland, llegando algunas de estas a poner el Don Sylvio incluso a la misma altura que el Tristram Shandy de STERne, así por ejemplo Wilson, 1981 (cf. PARAdA, 1997:193 ss.); HINDERER, 1993, caracteriza el Don Sylvio de una "ebenso geistreichen wie amüsanten Auseinandersetzung mit dem zeitgenössischen Phänomen der Schwärmerei» (HINDERER, 1993:185; "de un análisis tan ingenioso como divertido de esa exaltación arrobada tan propia de la época». Traducción mía, A.P.)
} 
Por el contrario, a partir de ahora y durante mucho tiempo, a la literatura alemana va a corresponderle la misión de intentar salvar el dualismo, cuasi metafísico, entre sujeto y objeto, razón y sentimiento, casualidad y necesidad: un dualismo, pues, efectivamente 'quijotesco'. El precio va a ser, en palabras de Siegfried J. Schmidt, la ««Entfremdung» von der unmittelbaren Lebenspraxis», el extrañamiento ante la 'realidad' o, si se prefiere un término más amplio, ante la vida. La lectura, asimilación y uso que se haga de bienes o herramientas culturales tenderá, en lo sucesivo, a paliar este estado de insuficiencia. El movimiento romántico, en sí, y la reinterpretación que los románticos alemanes hacen del Quijote serán buena muestra de ello (cf. Brüggemann, 1958).

\section{REFERENCIAS BIBLIOGRÁFICAS}

Blitz, Hans-Martin (2000): Aus Liebe zum Vaterland. Die deutsche Nation im 18. Jahrhundert, Hamburger Edition, Hamburg.

BRÜGgemanN, WeRnER (1958): Cervantes und die Figur des Don Quijote in Kunstanschauung und Dichtung der deutschen Romantik, Spanische Forschungen der Görresgesellschaft, Aschendorff.

Debus, Allen G. (1970): Science and Education in the Seventeenth Century. The Webster - Ward Debate, Macdonald, American Elsevier Inc., New York, London.

Elias, Norbert (1990): La sociedad de los individuos, Península, Barcelona. Traducción de José Antonio Alemany.

EVEN-ZOHAR, ITAMAR (1999): "La literatura como bienes y como herramientas», en: Villanueva, Darío/Monegal, Antonio/Bou, Eric (edts.): Sin fronteras. Ensayos de literatura comparada en homenaje a Claudio Guillén, Editorial Castalia, Madrid: 27-36.

GNISCI, ARMANDo (ed.) (2002): Introducción a la literatura comparada, Crítica, Barcelona. Traducción de Luigi Giuliani.

GRIMM, GUNTER E. (1983): Literatur und Gelehrtentum in Deutschland. Untersuchungen zum Wandel ihres Verhältnisses vom Humanismus bis zur Frühaufklärung, Max Niemeyer, Tübingen.

Guillén, Claudio (1995): El sol de los desterrados: literatura y exilio, Quaderns Crema, Sirmio, Barcelona.

HAUSER, ARNOLd (19883): Soziologie der Kunst, Beck, München.

HINDERER, WALTER (1993): "Christoph Martin Wieland», en: Grimm, Gunter E./ Max, Frank Rainer (eds.): Deutsche Dichter, Philipp Reclam jun., Stuttgart: 180-189.

JURT, JOSEPH (1997): «Bourdieus Analyse des literarischen Feldes oder der Universalitätsanspruch des sozialwissenschaftlichen Ansatzes», en: Internationales Archiv für Sozialgeschichte der deutschen Literatur, 22. Bd., 2. Heft:152-179.

MAHLER, ANDREAS (1991): "Jahrhundertwende, Epochenschwelle, epistemischer Bruch? England um 1600 und das Problem überkommener Epochenbegriffe», en: Garber, Klaus (ed.): Europäische Barockrezeption (2 tomos.), Wolfenbütteler Arbeiten zur Barockforschung, Wiesbaden, tomo 2: 995-1026.

NEUBERT, ALBRECHT (1999): «Übersetzungswissenschaft im Widerstreit. Äußere und innere Entwicklung einer Disziplin», en: Gil, Alberto/Steiner, Erich/GerzymischArbogast, Heidrun (eds.): Modelle der Translation. Grundlagen für Methodik, Bewertung, Computermodellierung, Peter Lang, F/M et al.: 11-32. 
NÜNNING, ANSGAR (ed.) (2001): Metzler Lexikon Literatur- und Kulturtheorie. Ansätze - Personen - Grundbegriffe. 2. überarbeitete und erweiterte Auflage, Metzler, Stuttgart- Weimar.

PARADA, ARTURO (2003): "Literatura y sociedad, individuo y comunidad: tres calas», en: PAATZ, ANNETTE/POHL, BURKHARD (eds.): Una sociología de la literatura para el siglo XXI/Literatursoziologie im 21. Jahrhundert, edition Tranvía-Verlag, Walter Frey, Berlín: 299-309.

- (1997): Offene literarische Welten gegen geschlossene Denkmodelle und Sozialsysteme: Don Quijote und Anton Reiser. Vervuert Verlag, Frankfurt am Main.

RAABE, MECHTHILD (1989): Leser und Lektüre im 18. Jahrhundert. Die Ausleihbücher der Herzog August Bibliothek Wolfenbüttel 1714-1799, (4 tomos), K.G. Saur, München-London-New York-Paris.

SWINGEWOOD, ALAN (1998): Cultural theory and the problem of modernity, St. Martin's Press, New York.

WILSON, DANIEL W. (1981): The narrative strategy of Wielands Don Sylvio von Rosalva, Peter Lang, Bern. 